\title{
Energy harvesting and information transmission scheme with UAV relay cooperation
}

\author{
Baofeng Ji ${ }^{1,2,3^{*}}$, Zhenzhen Chen ${ }^{1}$, Yuqi Li ${ }^{1}$, Sudan Chen ${ }^{4}$, Guoqiang Zheng ${ }^{1}$ and Hong Wen ${ }^{3}$
}

\begin{abstract}
This paper proposed a system architecture model of two-hop unmanned aerial vehicle (UAV) relay wireless communication and designed an energy harvesting and information transmission algorithm based on the energy harvested by UAV relay node. The energy of nodes except source node can be obtained by energy harvesting and all the UAV relay nodes transmitted signals via power splitting. Under the advance of non-static channel, the information user nodes were configured with multiple antennas and adopted max ratio combination (MRC). Based on the optimization criterion of energy efficiency maximization, the analytical solution of the optimal power allocation scheme for energy harvested and information transmission of multi-user two-UAV relay system was derived in detail. Since the optimization problem was a non-convex problem, this paper adopted the high signal-to-noise ratio approximation method and the power splitting method to realize the closed-form solution expression. The optimal solution of the objective function subjected with constraints can be obtained by Lagrangian algorithm and Lambert W function. Finally, the proposed algorithms and theoretical analysis are verified by simulations.
\end{abstract}

Keywords: Unmanned aerial vehicle, Relay cooperation, Energy harvesting, Optimization

\section{Introduction}

With the continuous growth of people's needs, the future wireless communication system needs better communication quality and wider communication range than the existing communication system [1]. In recent years, the concept of UAV communication is proposed in order to expand the network coverage in wireless communication. UAV communication is to use UAV as an air access point or a relay to increase network coverage and enhance link performance [2-5]. In addition, UAV communication can reduce the blocking of urban buildings, mountains, and other obstacles, so as to obtain higher LoS transmission effect $[6,7]$. At the same time, UAV has been widely used due to its advantages of strong mobility, flexible deployment, and low operating cost, including weather monitoring, forest fire detection, traffic control, cargo

*Correspondence: fengbaoji@126.com

'School of Information Engineering, Henan University of Science and Technology, Luoyang, China

${ }^{2}$ LAGEO, Institute of Atmospheric Physics, Chinese Academy of Sciences, Beijing, China

Full list of author information is available at the end of the article transportation, and emergency search [8]. UAV communication will become an important research direction in future communication system.

Due to its advantages, UAV has become one of the research hotspots in the field of communication [9]. At present, some literatures have studied about UAV communication, Yong Zeng and co-author proposed an energy-saving UAV communication system, which can improve the energy efficiency of the system by optimizing the trajectory of the UAV [10]. On this basis, Ladosz Pawel and co-author studied the trajectory planning scheme in the dynamic urban environment that improved the communication quality through the optimal positioning and trajectory planning algorithm [11]. Sekander Silvia and co-author considered the design and optimization of UAV network, which maximized the transmission rate by formulating subcarrier allocation and trajectory control [12]. However, the above literatures did not fully consider the existence of multiple types of nodes in the system. Although the system energy efficiency of UAV relay network has been optimized, many researches are 
quite different from this paper in terms of system model, objective function, and constraint conditions.

Although UAVs have many advantages, they are batterypowered devices whose endurance and power consumption limit their wider applications. RF energy harvesting technology can harvest energy from dedicated RF sources or signals in the surrounding environment $[13,14]$. Energy harvesting technology is introduced to alleviate the energy consumption problem of UAV equipment. The introduction of radio frequency (RF) energy harvesting in UAV relay has become a research hotspot. Meng Hua and Sixing Yin studied the AF UAV relay network and the typical single-source dual-target system, respectively. Their base stations are simultaneous wireless information and power transfer (SWIPT) of the UAV and maximizing throughput by using joint multi-parameters [15, 16]. Lifeng Xie and co-author proposed an uplinking transmission energy harvesting system of UAV, which uses wireless power transmission to charge the user, who used the harvested energy to send information to the UAV to realize the system communication process [17]. Further Sixing Yin and co-author considered an energy-constrained UAV cellular network, which ACTS as a relay and improved the uplink rate through cooperative communication among all users [18]. Weidang $\mathrm{Lu}$ and co-author proposed an energyconstrained UAV communication network protocol based on orthogonal frequency division multiple access (OFDM) relay wireless power transmission [19]. This paper applies energy harvesting technology to UAV to alleviate energy consumption of UAV equipment. However, most of them are designed to solve the problem of energy consumption of UAV, which is less considered for subordinate users. This paper not only uses energy harvesting technology to alleviate the problem of UAV energy consumption, but also uses the characteristics of UAV mobility to charge the subordinate users.

The structure of this paper is as follows: Section 2 describes the methods used in the paper. Section 3 introduces the system model of UAV relay auxiliary communication. The optimal transmitting power is deduced in Section 4. Section 5 simulates and verifies the proposed scheme and theoretical analysis. Section 6 draws a conclusion in the paper.

\section{Methods}

This paper mainly studies the cooperative energy harvesting and information transmission algorithm of UAV relay, which is mainly reflected in the wide coverage and lowpower scenario of UAV relay node cooperation. Among them, wide coverage is mainly reflected in the cooperative transmission and energy harvesting of UAV relay nodes, while low power consumption is mainly reflected in the algorithm design of precoding or beamforming at the sending end of this paper. Under the advance of non-static channel, the information user nodes were configured with multiple antennas and adopted max ratio combination (MRC). Moreover, considering the actual power conversion efficiency and the power consumption factor of the hardware circuit loss in the EARTH plan [20], the analytical solution of the optimal power allocation scheme for energy harvested and information transmission of multi-user UAV two-relay system was derived in detail. In addition, the asymptotically optimal transmission power solution is the minimum transmission power, so that the nodes of the system have lower power consumption and can realize a greener wireless communication transmission environment.

\section{System model}

As shown in Fig. 1, the system model described the energy harvested and information transmission of the multi-hop wireless network. It is worth noting that the other nodes harvest energy from the previous nodes in order to solve the energy-constrained problem. Moreover, the UAV is a battery-powered device, and it is often difficult to replace the battery of the drone directly, so the assisted UAV relay node uses the previous node to receive the required information while harvesting energy via wireless channel. Therefore, in the first hop phase, the first UAV relay node $R_{1}$ harvests energy and receives information from the source node. In the second hop phase, the first UAV relay node $R_{1}$ uses the harvested energy to transmit the signal to the second UAV relay node $R_{2}$ and the harvesting energy node. In the third hop phase, the second UAV relay node $R_{2}$ uses the harvested energy to transmit the information to the receiving information node.

In the first hop phase, the signal received by the UAV relay node $R_{1}$ can be given by:

$$
y_{R 1}=\sqrt{P_{s}}(\mathbf{h}+\Delta \mathbf{h}) x+n_{1}
$$

where $P_{s}$ is the transmit power of the source node in the network and the transmit power of the UAV relay node $R_{1}$ is the energy obtained by the signal sent by the source node. The signal transmission of the UAV relay node $R_{1}$ for the second hop is completely dependent on the energy acquired by the signal of the source node. So, it is necessary to optimize the information transmission and energy harvested of the transmit power of the source node, where $\mathbf{h} \sim C N(0,1)$ is the wireless channel from the source to the UAV relay node, subject to a cyclic symmetric complex Gaussian distribution with zero mean and one variance, and $\Delta \mathbf{h}$ is the jitter wireless channel from the transmitting source node to the UAV relay node $\mathrm{R}_{1} . x$ is the transmitted signal and $n_{1} \sim C N\left(0, \sigma_{1}^{2}\right)$ is the additive white Gaussian noise (AWGN) at the UAV relay node $\mathrm{R}_{1}$.

After receiving the signal from the source node, the UAV relay node $R_{1}$ uses the power splitting method to harvest energy as: 


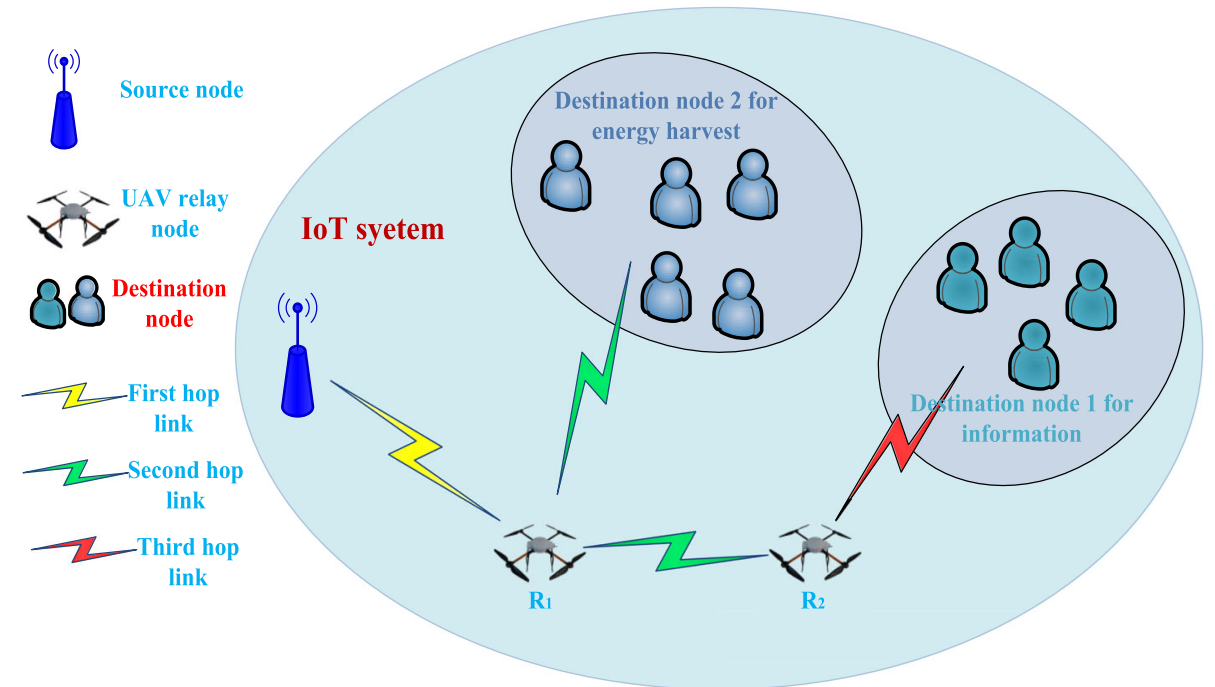

Fig. 1 The system model of energy and information wireless transmission with UAV relay coordination

$$
y_{R_{1, E}}=\sqrt{1-\rho} y_{R_{1}}
$$

where $0<\rho<1$ is the power splitting factor, so the energy after the UAV relay node separates the signal from all received signals is $\sqrt{1-\rho}$. The energy harvested is:

$$
P_{E_{1}}=(1-\rho)\left(P_{s}\|\mathbf{h}+\mathbf{h}\|^{2}+\sigma_{1}^{2}\right)
$$

After the power splitting, the wireless signal received by the UAV relay node $R_{1}$ is:

$$
y_{R_{1, I}}=\sqrt{\rho} y_{R_{1}}+z_{1}
$$

where $z_{1} \sim C N\left(0, \delta_{z 1}^{2}\right)$ is the conversion noise from the radio frequency to the baseband.

The power of the signal received by the UAV relay node $\mathrm{R}_{1}$ is:

$$
P_{I_{1}}=\rho\left(P_{s}\|\mathbf{h}+\Delta \mathbf{h}\|^{2}+\sigma_{1}^{2}\right)+\sigma_{z_{1}}^{2}
$$

The signal transmitted by the UAV relay node $R_{1}$ is:

$$
\begin{aligned}
& \tilde{y}_{R_{1}}=\sqrt{\frac{\| y_{R_{1, E} \|_{F}}^{2}}{\left\|y_{R_{1, I}}\right\|_{F}^{2}} y_{R_{1}}} \\
& =\sqrt{\frac{(1-\rho)\left(P_{s}\|\mathbf{h}+\Delta \mathbf{h}\|^{2}+\sigma_{1}^{2}\right)}{\rho\left(P_{s}\|\mathbf{h}+\Delta \mathbf{h}\|^{2}+\sigma_{1}^{2}\right)+\sigma_{z_{1}}^{2}}} \cdot\left(\sqrt{P_{s}}(\mathbf{h}+\Delta \mathbf{h}) x+n_{1}\right)
\end{aligned}
$$

In the second hop phase, the energy harvested by the destination node for energy harvesting is:

$$
\left\|y_{E}\right\|^{2}=\sigma_{E}^{2}+\|\mathbf{k}+\Delta \mathbf{k}\|^{2}(1-\rho)\left(P_{s}\|\mathbf{h}+\Delta \mathbf{h}\|^{2}+\sigma_{1}^{2}\right)
$$

where $\mathbf{k} \sim C N(0,1)$ is wireless channel from the UAV relay node $R_{1}$ to the harvested energy node, $\Delta \mathbf{k}$ is a jitter wireless channel from the UAV relay node $R_{1}$ to the harvested energy node, and $\sigma_{E}^{2}$ is the variance of the noise at the harvested energy node. In addition, the signal received by the UAV relay node $R_{2}$ is:

$$
y_{R_{2}}=(\mathbf{g}+\Delta \mathbf{g}) \cdot \tilde{y}_{R_{1}}+n_{2}
$$

where $\mathbf{g} \sim C N(0,1)$ is the wireless channel from the UAV relay node $R_{1}$ to the UAV relay node $R_{2}, n_{2}$ is jitter wireless channel from the UAV relay node $R_{1}$ to the UAV relay node $\mathrm{R}_{2}$, and $n_{2}$ is the AWGN noise at the UAV relay nod $\mathrm{R}_{2}$, where $n_{2} \sim C N\left(0, \sigma_{2}^{2}\right)$. The UAV relay node $\mathrm{R}_{2}$ continues to adopt the power splitting method (the factor is $\theta$ ) and the received energy and signals can be expressed as:

$$
\begin{aligned}
& y_{R_{2, E}}=\sqrt{1-\theta} y_{R_{2}} \\
& y_{R_{2, I}}=\sqrt{\theta} y_{R_{2}}+z_{2}
\end{aligned}
$$

Then the signal sent by the UAV relay node $R_{2}$ is:

$$
\begin{aligned}
& \tilde{y}_{R_{2}}=\sqrt{\frac{\left\|y_{R_{2, E}}\right\|_{F}^{2}}{\left\|y_{R_{2, I}}\right\|_{F}^{2}} y_{R_{2}}} \\
& =\sqrt{\frac{(1-\theta)\|\mathbf{g}+\mathbf{g}\|^{2}(1-\rho)\left(P_{s}\|\mathbf{h}+\mathbf{h}\|^{2}+\sigma_{1}^{2}\right)^{2}+(1-\theta) \sigma_{2}^{2}\left[\rho\left(P_{s}\|\mathbf{h}+\mathbf{h}\|^{2}+\sigma_{1}^{2}\right)+\sigma_{z_{1}}^{2}\right]}{\theta\|\mathbf{g}+\mathbf{g}\|^{2}(1-\rho)\left(P_{s}\|\mathbf{h}+\mathbf{h}\|^{2}+\sigma_{1}^{2}\right)^{2}+\left(\theta \sigma_{2}^{2}+\sigma_{z 2}^{2}\right)\left[\rho\left(P_{s}\|\mathbf{h}+\mathbf{h}\|^{2}+\sigma_{1}^{2}\right)+\sigma_{z_{1}}^{2}\right]}} \\
& \cdot\left[(\mathbf{g}+\mathbf{g}) \cdot \sqrt{\frac{(1-\rho)\left(P_{s}\|\mathbf{h}+\mathbf{h}\|^{2}+\sigma_{1}^{2}\right)}{\rho\left(P_{s}\|\mathbf{h}+\mathbf{h}\|^{2}+\sigma_{1}^{2}\right)+\sigma_{z_{1}}^{2}}}\left(\sqrt{P_{s}}(\mathbf{h}+\mathbf{h}) x+n_{1}\right)+n_{2}\right]
\end{aligned}
$$

After the third hop transmission, the signal received by the destination node can be given as:

$$
y_{D}=w\left[(f+\Delta f) \tilde{y}_{R_{2}}+n_{3}\right]
$$

where $w$ is prereception matrix and equals to $\frac{f^{H}}{\|f\|}$, $f \sim C N(0, m)$ is wireless channel from the UAV relay node $\mathrm{R}_{2}$ to the destination node of the received signal, $\Delta f$ is jitter wireless channel from the UAV relay node $R_{2}$ to the destination node of the received signal, and 
$n_{3}$ is the AWGN noise at the destination node, where $n_{3} \sim C N\left(0, \sigma_{3}^{2}\right)$.

After substituting Eq. (11) into Eq. (12):

$y_{D}=w\left\{\begin{array}{l}(f+\Delta f) \cdot \sqrt{\frac{(1-\theta)\|\mathbf{g}+\mathbf{g}\|^{2}(1-\rho) A^{2}+(1-\theta) \sigma_{2}^{2}\left(\rho A+\sigma_{z_{1}}^{2}\right)}{\theta\|\mathbf{g}+\mathbf{g}\|^{2}(1-\rho) A^{2}+\left(\theta \sigma_{2}^{2}+\sigma_{z_{2}}^{2}\right)\left(\rho A+\sigma_{z_{1}}^{2}\right)}} \\ \cdot\left[\mathbf{g} \cdot \sqrt{\frac{(1-\rho) A}{\rho A+\sigma_{z_{1}}^{2}}}\left(\sqrt{P_{s}} \mathbf{h} x+n_{1}\right)+n_{2}\right]+n_{3}\end{array}\right\}$

where $A=P_{s}\|\mathbf{h}+\mathbf{h}\|^{2}+\sigma_{1}^{2}$.

\section{Asymptotically optimal algorithm}

This section mainly solves the optimal transmit power at the source. Therefore, this part designs precoding algorithm at source node to achieve green communications. The transmit power of source node can be optimized to meet the required energy by the energy harvested node to improve the energy efficiency of the entire system. The energy efficiency function is defined as the ratio of the instantaneous throughput to the total power consumption of the hardware circuit loss.

From (13), it can be derived that the SNR at the received signal node after three-hop transmission:

$$
S N R_{D}=\frac{\|\mathbf{g}+\Delta \mathbf{g}\|^{2}\|\mathbf{h}+\Delta \mathbf{h}\|^{2} P_{s}(1-\rho) A}{\left[\|\mathbf{g}+\Delta \mathbf{g}\|^{2} \sigma_{1}^{2}(1-\rho) A+\sigma_{2}^{2}\left(\rho A+\sigma_{z_{1}}^{2}\right)\right]+}
$$

Based on Eqs. (14) and (7), the minimum energy required can be constrained and the optimization problem can be expressed as:

$$
\begin{aligned}
& \max _{P_{s}} \frac{\log _{2}\left(1+S N R_{D}\right)}{a P_{s}+b m}, \\
& \text { s.t. } \sigma_{E}^{2}+\|\mathbf{k}+\Delta \mathbf{k}\|^{2}(1-\rho)\left(P_{s}\|\mathbf{h}+\Delta \mathbf{h}\|^{2}+\sigma_{1}^{2}\right) \geq \alpha_{0}
\end{aligned}
$$

where $\alpha_{0}>0$ is the preset threshold, which defines the minimum energy harvested at the destination node. $a>0$ and $b>0$ are parameters in the power consumption model that consider power conversion efficiency and hardware circuit power consumption cost, and the model is widely used in the EARTH project.

The purpose of this section is to derive optimal analytical expression of the source transmit power. However, the objective function in Eq. (15) can be seen that $P_{s}$ is a cubic term, so the calculation can be very difficult. In order to solve this problem, a high SNR approximation method can be used to approximate the SNR in Eq. (14). In high SNR, the noise variance $\sigma_{z}^{2}$ can be ignored. Using a high SNR approximation and the average allocation strategy for the power of the UAV relay node $\mathrm{R}_{2}$, Eq. (14) can be simplified as follows:
$S N R_{D}{ }^{\prime}=P_{s} \frac{\|\mathbf{g}+\Delta \mathbf{g}\|^{2}(1-\rho)\|\mathbf{h}+\Delta \mathbf{h}\|^{2}}{\|\mathbf{g}+\Delta \mathbf{g}\|^{2}(1-\rho) \sigma_{1}^{2}+\rho \sigma_{2}^{2}+\frac{\sigma_{3}^{2} \rho}{\|w(f+\Delta f)\|^{2}}} \triangleq \varpi P_{s}$

Substituting Eq. (16) into Eq.(15) , the problem becomes:

$\max _{P_{s}} \frac{\log _{2}\left(1+S N R_{D}{ }^{\prime}\right)}{a P_{s}+b m}$,

s.t. $\sigma_{E}^{2}+\|\mathbf{k}+\Delta \mathbf{k}\|^{2}(1-\rho)\left(P_{s}\|\mathbf{h}+\Delta \mathbf{h}\|^{2}+\sigma_{1}^{2}\right) \geq \alpha_{0}$

Equation (17) can be expressed as (18) to facilitate to solve with the Lagrangian multiplier method:

$$
\begin{aligned}
& \min _{P_{S}} \frac{a P_{s}+b m}{\log _{2}\left(1+\operatorname{SNR}_{D}{ }^{\prime}\right)} \\
& \text { s.t. } P_{S} \geq \frac{\alpha_{0}-\sigma_{E}^{2}}{\|\mathbf{h}+\Delta \mathbf{h}\|^{2}\left(\|\mathbf{k}+\Delta \mathbf{k}\|^{2}(1-\rho)\right)}-\frac{\sigma_{1}^{2}}{\|\mathbf{h}+\Delta \mathbf{h}\|^{2}} \triangleq \varphi
\end{aligned}
$$

The constraint can be written as:

$$
\frac{1}{\log _{2}\left(1+\varpi P_{s}\right)} \leq \frac{1}{\log _{2}(1+\varpi \varphi)}
$$

Substituting (19) into Eq. (18):

$$
\begin{aligned}
& \min _{P_{s}} \frac{a P_{s}+b m}{\log _{2}\left(1+\mathrm{SNR}_{D^{\prime}}\right)} \\
& \text { s.t. } \frac{1}{\log _{2}\left(1+\varpi P_{s}\right)} \leq \frac{1}{\log _{2}(1+\varpi \varphi)}
\end{aligned}
$$

Express (20) as a Lagrangian function:

$\tilde{\phi}=\frac{a P_{s}+b m}{\log _{2}\left(1+\varpi P_{s}\right)}+\lambda\left\{\frac{1}{\log _{2}\left(1+\varpi P_{s}\right)}-\frac{1}{\log _{2}(1+\varpi \varphi)}\right\}$

where $\lambda$ is the Lagrange multiplier.

By finding the first derivative of $P_{s}$ and making it zero, the optimal solution is:

$\frac{\partial \tilde{\phi}}{\partial P_{s}}=\frac{a \log _{2}\left(1+\varpi P_{s}\right)-\left(a P_{s}+b m\right) \frac{\omega}{\ln 2\left(1+\varpi P_{s}\right)}-\lambda \frac{\varpi}{\ln 2\left(1+\varpi P_{s}\right)}}{\left[\log _{2}\left(1+\varpi P_{s}\right)\right]^{2}}=0$

Thus, the optimal solution to (22) is obtained as:

$$
\begin{aligned}
& \ln 2 \frac{1}{1+\varpi P_{s}} \\
& =W\left\{\ln 2 \frac{2^{\ln 2}}{a} a \frac{1}{\varpi \lambda+\frac{1}{\ln 2}(b m \varpi-a)}\right\} \\
& =W\left\{2^{\ln 2} \frac{1}{\ln 2 \varpi \lambda+b m \varpi-a}\right\}
\end{aligned}
$$

where $\mathrm{W}\{\cdot\}$ is the Lambert function [21], so that the asymptotically optimal transmit power of the source node can be expressed as:

$$
P_{s}=\left(\frac{\ln 2}{W\left\{2^{\ln 2} \frac{1}{\ln 2 \varpi \lambda+b m \varpi-a}\right\}}-1\right) \frac{1}{\varpi}
$$

Therefore, through the above derivation and analysis, the optimal source transmit power when the received energy and information node coexist in the multi-hop system are obtained. 


\section{Results and discussion}

This section simulates and analyzes the energy efficiency of the system implemented by the scheme proposed in this paper. The simulation tool uses MATLAB, and the number of Monte Carlo cycles is 10000. Both the harvested energy node and the information node receive energy and information through wireless channel. The parameter $a$ in the power consumption model $\left(p_{\text {total }}=a p_{t}+b\right)[22]$ ranges from 2 to 10 , and $\mathrm{b}$ ranges from 2 to 10 . All noise variances are set to 1 , such as $\sigma_{1}^{2}=\sigma_{2}^{2}=\sigma_{3}^{2}=\sigma_{E}^{2}=\sigma_{z_{1}}^{2}=\sigma_{z_{2}}^{2}=1$, the channel follows Rayleigh fading distribution.

\subsection{Results}

Figure 2 shows the energy efficiency of the entire system for the power splitting factor $\rho=0.5$. It can be seen from the figure that for any parameter value of the power consumption model, the energy efficiency tends to be convex with the parameter $\alpha_{0}$ increasing. This is because the throughput gain is much greater than the power consumption at low $\alpha_{0}$, which indicates that the energy efficiency of the entire system is improved. However, the throughput gain is limited with increased power for high $\alpha_{0}$. It can be seen from Fig. 2, for a given $b$, as the parameter $a$ increases, the corresponding $\alpha_{0}$ value can be decreased.

Figures 3 and 4 show that the energy efficiency function of the entire system for the power splitting factor $\rho=0.1$ and $\rho=0.9$. Similar to Fig. 2, the energy efficiency is a convex function. As can be seen from Fig. 3, when the power splitting factor $\rho$ is 0.1 , the system energy efficiency may be increased compared to $\rho$ equals to 0.5 . This is because when the power splitting factor $\rho$ is small, the system has a weak ability to harvest energy, the efficiency of receiving information is high, which may result in a decrease in system energy efficiency. Similarly, as can be seen from Fig. 4, when the value of the power splitting factor $\rho$ is large, the system has a weak ability to transmit information, but the ability to harvest energy will be increased, so the energy efficiency of system is also low. Therefore, the appropriate value of power splitting factor $\rho$ plays an important role in improving the system energy efficiency.

Figure 5 depicts the energy efficiency of system when the power consumption parameter $a=5, b=10,[20]$ and the power splitting factor $\rho$ set $0.2,0.3$, and 0.7 , respectively. As can be seen from the Fig. 5, the energy efficiency function shows different trends with the change of $\rho$ value. This is because the $\rho$ value determines both the harvested energy and the transmitted signal of the system. Therefore, the joint optimization of the transmit power and the power splitting factor $\rho$ at the source node will be the next research topic.

In this paper, due to the optimization problem is a non-convex problem, the asymptotically optimal solution of the optimization problem is obtained by the high SNR approximation method and Lagrangian algorithm and Lambert $\mathrm{W}$ function in order to solve the objective

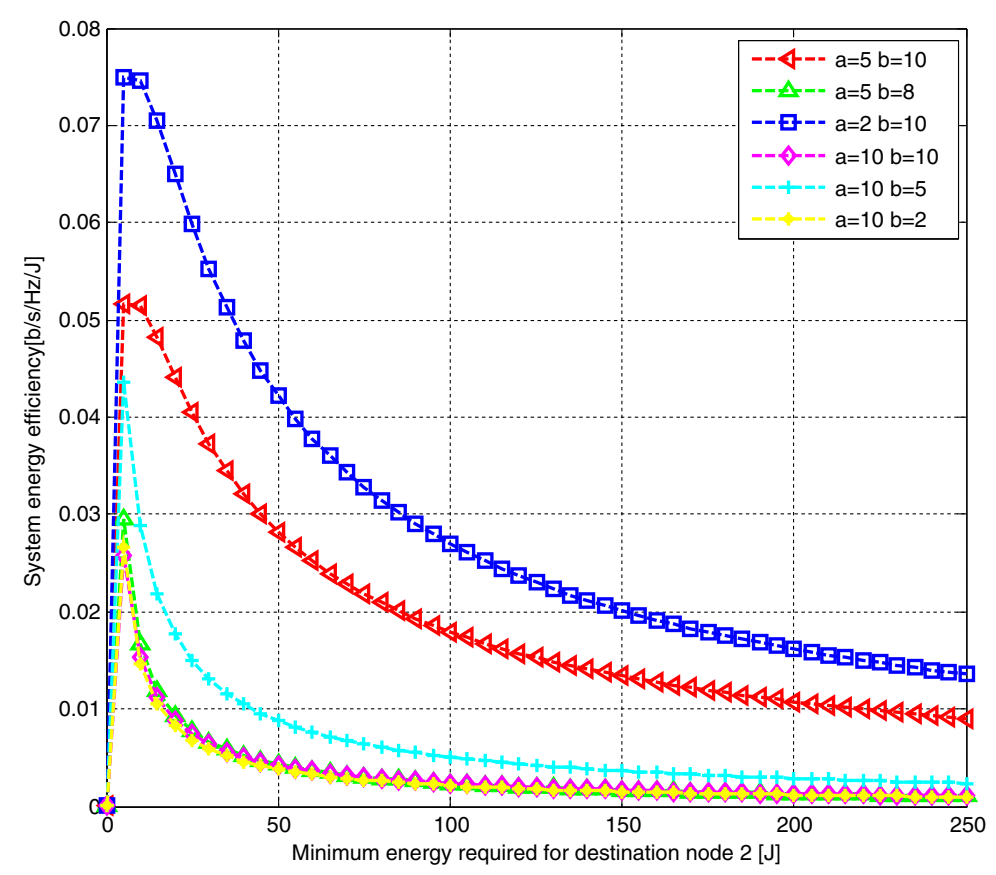

Fig. 2 Achieved energy efficiency of the whole loT systems by the proposed scheme at the power splitting factor $\rho=0.5$ 


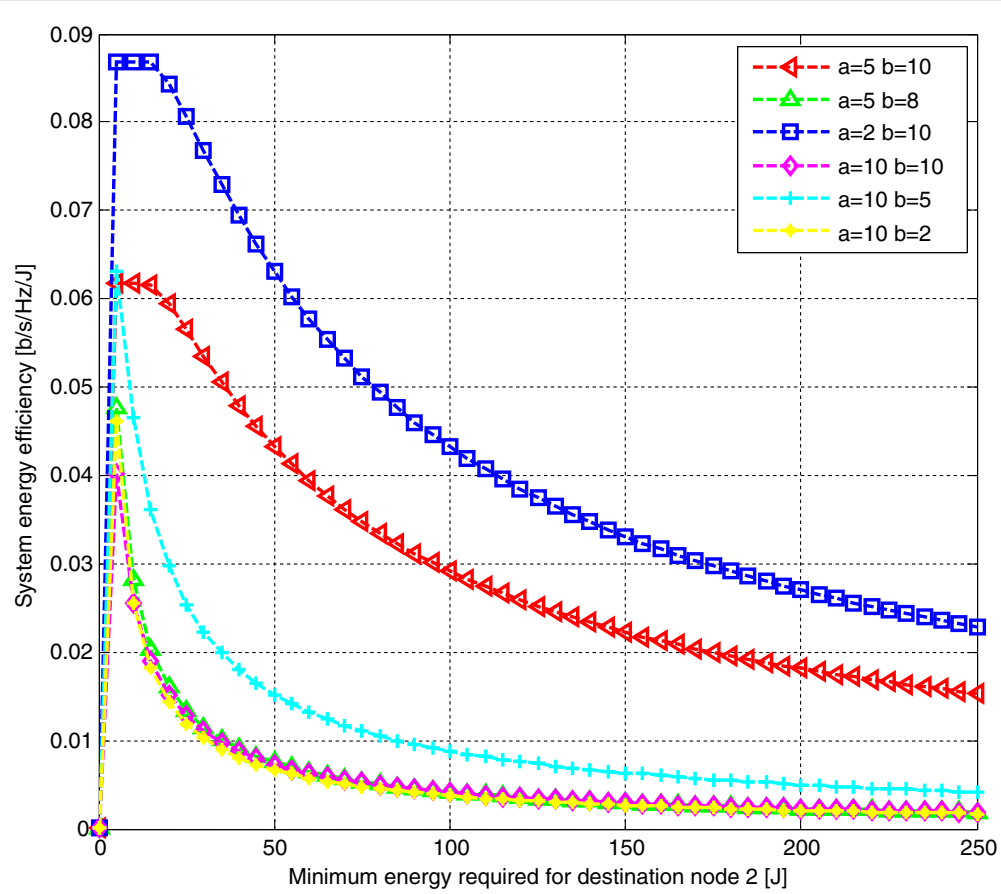

Fig. 3 Achieved energy efficiency of the whole loT systems by the proposed scheme at the power splitting factor $\rho=0.1$

function. Additionally, comparing the difference between the approximate and the original solution, Fig. $6 \mathrm{com}-$ pares and analyzes the asymptotically optimal solution with the original optimization problem. It can be seen from Fig. 6 that the asymptotically optimal solution solved in the paper and the optimal solution of the original optimization problem almost coincide in the multi-energy harvested region, where the difference is small. Therefore, the asymptotically optimal solution can be used as a simple method to solve the optimization problem.

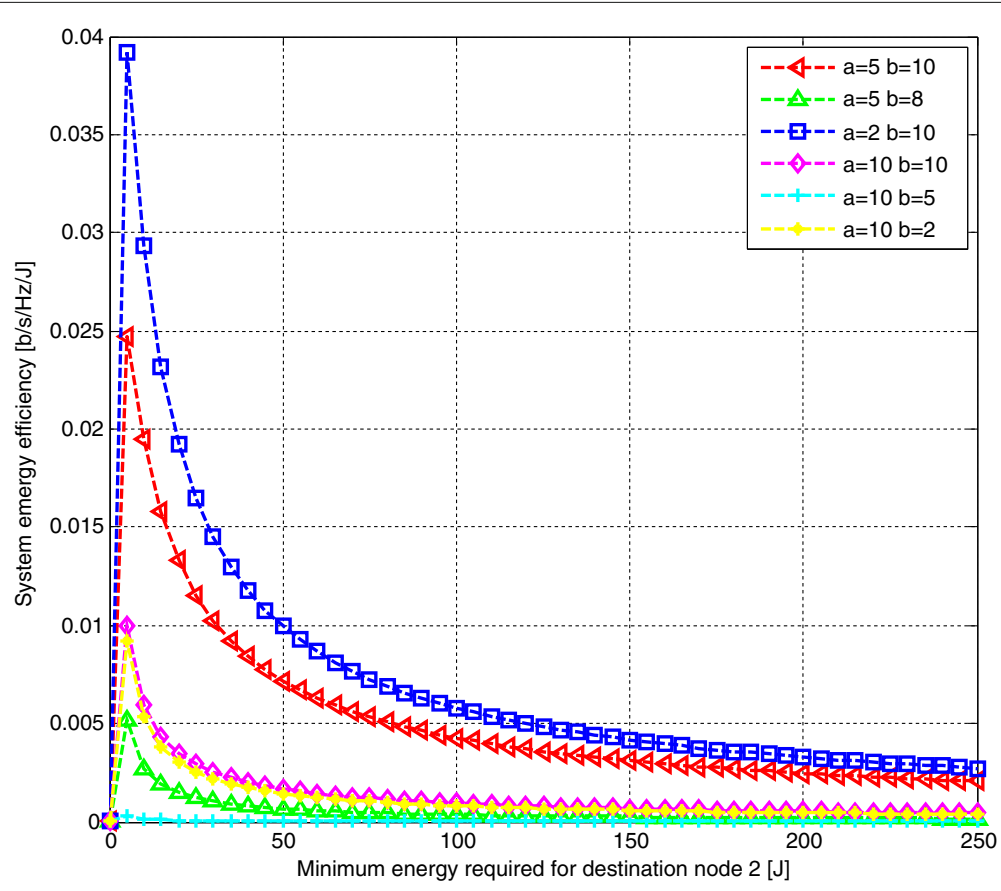

Fig. 4 Achieved energy efficiency of the whole loT systems by the proposed scheme at the power splitting factor $\rho=0.9$ 


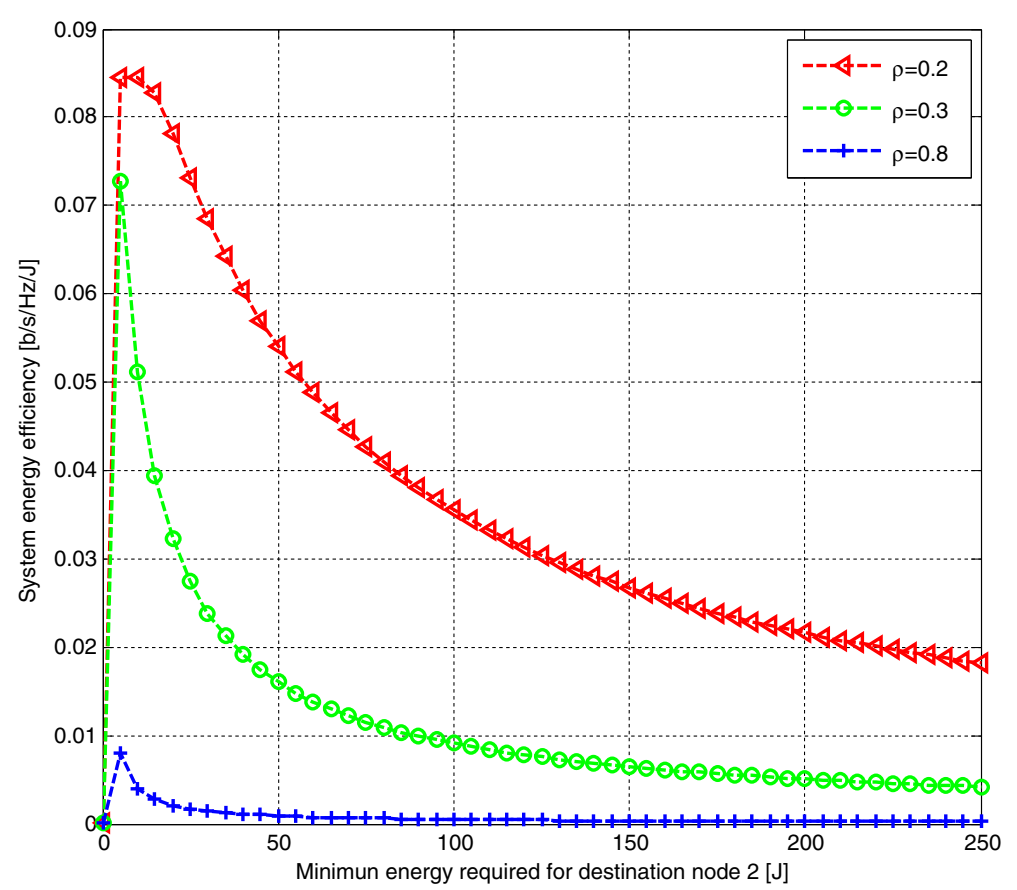

Fig. 5 Achieved energy efficiency of the whole loT systems by the proposed scheme at the different power splitting factor $\rho$ with $a=5, b=10$

\subsection{Discussion}

Compared with existing literatures, the proposed scheme was based on non-static wireless channel, which was equipped with multiple antennas at the destination end and adopted the multi-hop transmission scheme prereceived by MRC to increase the transmission range and reduces the energy consumption. However, there are still some shortages in our proposed transmission scheme. First of all, as can be seen from the simulation results, the power splitting factor has an impact on the energy efficiency of the system, and the optimal power splitting factor has not been optimized in this paper. In addition,

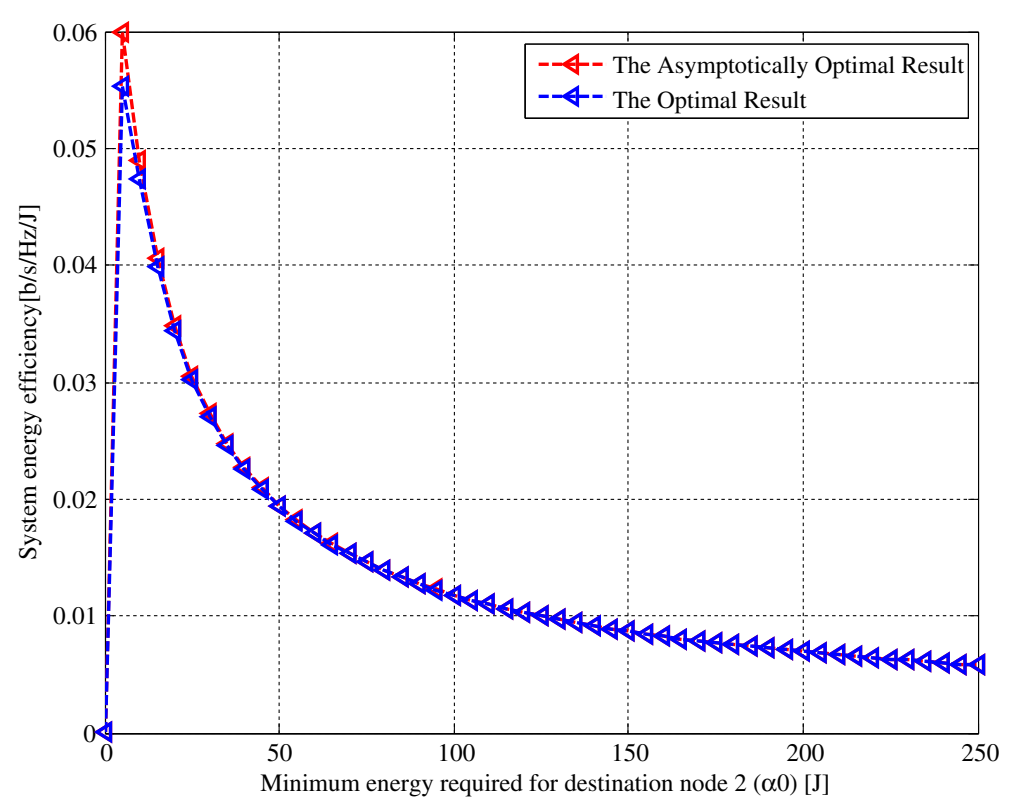

Fig. 6 The comparison between the asymptotically optimal result and the globally optimal result 
although the proposed scheme considers the situation of multiple antennas for the purpose, there is no joint optimization of the optimal number of antennas. Finally, in the future, multiple parameters such as the transmission power, power splitting factor, and the number of receiving antennas will be considered for joint optimization to make the proposed scheme more comprehensive.

\section{Conclusion}

In this paper, the energy harvested and information transmission scheme was designed for multi-hop communication system. The UAV relay node used the power splitting method for information transmission. Under the advance of non-static channel, the information user nodes were configured with multiple antennas and adopted max ratio combination (MRC). The energy efficiency function is defined as the ratio of instantaneous throughput to the total power consumption of the hardware circuit. The UAV relay node uses the harvested energy for information transmission. Considering the actual power conversion efficiency and the power consumption factor of the hardware circuit loss in the EARTH project, the analytical solution of the optimal power allocation scheme for the energy harvested and information transmission of wireless system is derived in closed-form expression. Since the optimization problem is difficult to calculate, the paper obtains the optimal solution of the optimization problem by using the high SNR approximation method. Finally, the system energy efficiency are verified through simulation. By comparing and analyzing the asymptotically optimal solution and the original optimization problem, it is proved that the asymptotic solution can be used as a simple method to solve the optimization problem. The important role of the power splitting factor can be seen through simulation. Therefore, the joint optimization of the source transmit power, power split factor, and UAV relay node selection will be the next research topic.

\section{Abbreviations}

AF: Audio frequency; OFDM: Orthogonal frequency division multiple access; SWIPT: Simultaneous wireless information and power transfer; UAVs: Unmanned aerial vehicles

\section{Acknowledgements}

Not applicable.

\section{Authors' contributions}

BJ conceived and designed the methods. BJ and ZC performed the experiments and completed the manuscript. YL and SC collated and analyzed The experimental data. GZ and HW gave valuable suggestions on the structure of the paper. All authors read and approved the final manuscript.

\section{Funding}

This work was supported by the National Thirteen Five Equipment Research Fund under Grant 6140311030207; National Natural Science Foundation of China under Grant 61801170, 61901241, 61671144, 61701172, 61902041; National Key Research and Development Plan under Grant 2018YFB0904905; Project of Education Department Cooperation Cultivation under Grant
201602011005 and 201702135098; China Postdoctoral Science Foundation under Grant 2018M633351; LAGEO of Chinese Academy of Sciences under Grant LAGEO-2019-2; Key Technology Project of Henan Province under Grant 1821021 10401; Program for Science \& Technology Innovation Talents in the University of Henan Province(2OHASTIT022, 17HASTIT025); 21 th Project of Xizang Cultural Inheritance and Development Collaborative Innovation Center in 2018; Natural Science Foundation of Xizang Named "Research of Key Technology of Millimeter Wave MIMO Secure Transmission with Relay Ehancement" in 2018; Xizang Autonomous Region Education Science "13th Five-year Plan" Major Project for 2018 (XZJKY201803) ; Young Backbone Teachers in Henan Province.

\section{Availability of data and materials}

The authors confirm that the data supporting the findings of this study are available within the article. All the simulation parameters and other experimental details are clearly shown in this manuscript, which could be used to interpret and replicate the findings reported in the article.

\section{Competing interests}

The authors declare that they have no competing interests.

\section{Author details}

${ }^{1}$ School of Information Engineering, Henan University of Science and Technology, Luoyang, China. ${ }^{2}$ LAGEO, Institute of Atmospheric Physics, Chinese Academy of Sciences, Beijing, China. ${ }^{3}$ School of Aeronautics and Astronautics, University of Electronic Science and Technology of China, Chengdu, China. ${ }^{4}$ College of Forestry, Henan University of Science and Technology, Kaiyuan avenue, Luoyang, People's Republic of China.

Received: 26 August 2019 Accepted: 11 November 2019

Published online: 19 December 2019

\section{References}

1. X. Liu, N. Ansari, Green relay assisted D2D communications with dual batteries in heterogeneous cellular networks for IOT. IEEE Internet Things J. 4(5), 1707-1715 (2017)

2. C. Li, H. J. Yang, F. Sun, J. M. Cioffi, L. Yang, Multiuser overhearing for cooperative two-way multiantenna relays. IEEE Trans. Veh. Technol. 65(5), 3796-3802 (2015)

3. C. Li, S. Zhang, P. Liu, F. Sun, J. M. Cioffi, L. Yang, Overhearing protocol design exploiting intercell interference in cooperative green networks. IEEE Trans. Veh. Technol. 65(1), 441-446 (2015)

4. K. Song, B. Ji, Y. Huang, M. Xiao, L. Yang, Performance analysis of heterogeneous networks with interference cancellation. IEEE Trans. Veh. Technol. 66(8), 6969-6981 (2017)

5. D. Cao, Y. Liu, X. Ma, J. Wang, B. Ji, C. Feng, J. Si, A relay-node selection on curve road in vehicular networks. IEEE Access. 7, 12714-12728 (2019)

6. Y. Xu, L. Xiao, D. Yang, L. Cuthbert, Y. Wang, Energy-efficient UAV communication with multiple GTS based on trajectory optimization. Mob Inf. Syst. 2018, 1-10 (2018)

7. S. Zhang, H. Zhang, B. Di, L. Song, Cellular UAV-to-X communications: design and optimization for multi-UAV networks. IEEE Trans. Wirel. Commun. 18(2), 1346-1359 (2019)

8. L. Gupta, R. Jain, G. Vaszkun, Survey of important issues in UAV communication networks. IEEE Commun. Surv. Tutor. 18(2), 1123-1152 (2015)

9. S. Arabi, E. Sabir, H. Elbiaze, M. Sadik, Data gathering and energy transfer dilemma in UAV-assisted flying access network for IOT. Sensors. 18(5), 1519 (2018)

10. Y. Zeng, R. Zhang, Energy-efficient uav communication with trajectory optimization. IEEE Trans. Wirel. Commun. 16(6), 3747-3760 (2017)

11. P. Ladosz, H. Oh, W.-H. Chen, Trajectory planning for communication relay unmanned aerial vehicles in urban dynamic environments. J. Intell. Robot. Syst. 89(1-2), 7-25 (2018)

12. S. Sekander, H. Tabassum, E. Hossain, Multi-tier drone architecture for 5G/B5G cellular networks: challenges, trends, and prospects. IEEE Commun. Mag. 56(3), 96-103 (2018)

13. C. Li, Y. Li, K. Song, L. Yang, Energy efficient design for multiuser downlink energy and uplink information transfer in 5G. Sci. China Inf. Sci. 59(2), 1-8 (2016) 
14. K. Song, B. Ji, C. Li, L. Yang, Outage analysis for simultaneous wireless information and power transfer in dual-hop relaying networks. Wirel. Netw. 25(2), 837-844 (2019)

15. M. Hua, C. Li, Y. Huang, L. Yang, in 2017 9th International Conference on Wireless Communications and Signal Processing (WCSP). Throughput maximization for UAV-enabled wireless power transfer in relaying system (IEEE, 2017), pp. 1-5. https://doi.org/10.1109/wcsp.2017.8170970

16. S. Yin, Y. Zhao, L. Li, in 2018 IEEE International Conference on Communications (ICC). UAV-assisted cooperative communications with time-sharing SWIPT (IEEE, 2018), pp. 1-6. https://doi.org/10.1109/icc.2018 8422536

17. L. Xie, J. Xu, R. Zhang, Throughput maximization for UAV-enabled wireless powered communication networks. IEEE Internet Things J. 6(2), 1690-1703 (2018)

18. S. Yin, Z. Qu, L. Li, in 2018 IEEE 87th Vehicular Technology Conference (VTC Spring). Uplink resource allocation in cellular networks with energy-constrained UAV relay (IEEE, 2018), pp. 1-5. https://doi.org/10 1109/vtcspring.2018.8417737

19. W. Lu, S. Fang, Y. Gong, L. Qian, X. Liu, J. Hua, in 2018 IEEE International Conference on Communications Workshops (ICC Workshops). Resource allocation for OFDM relaying wireless power transfer based energy-constrained UAV communication network (IEEE, 2018), pp. 1-6. https://doi.org/10.1109/iccw.2018.8403627

20. G. Auer, O. Blume, V. Giannini, I. Godor, M. Imran, Y. Jading, E. Katranaras, M. Olsson, D. Sabella, P. Skillermark, et al., Energy efficiency analysis of the reference systems, areas of improvements and target breakdown. Earth. 20(10) (2010)

21. S. R. Valluri, D. J. Jeffrey, R. M. Corless, Some applications of the lambert w function to physics. Can. J. Phys. 78(9), 823-831 (2000)

22. B. Ji, Z. Chen, S. Chen, B. Zhou, C. Li, H. Wen, Joint optimization for ambient backscatter communication system with energy harvesting for IoT. Mech. Syst. Signal. Process. 135, 106412 (2020)

\section{Publisher's Note}

Springer Nature remains neutral with regard to jurisdictional claims in published maps and institutional affiliations.

\section{Submit your manuscript to a SpringerOpen ${ }^{\circ}$ journal and benefit from:}

- Convenient online submission

- Rigorous peer review

- Open access: articles freely available online

- High visibility within the field

- Retaining the copyright to your article

Submit your next manuscript at $\gg$ springeropen.com 